\title{
MEDICAL NEWS FROM SCIENTIFIC ANALYSIS OF THE TURIN SHROUD
}

\author{
M. Bevilacqua', M. D’Arienzo ${ }^{2}$ \\ ${ }^{1}$ Hospital-University of Padua, Italy, matteo.bevilacqua@alice.it \\ ${ }^{2}$ Orthopaedic Clinic, University of Palermo, Italy, Michele.darienzo@unipa.it
}

\begin{abstract}
This paper synthetizes a series of works recently published in reference to medical studies regarding both the physical conditions of the Man who was wrapped in the Turin Shroud (TS) and the tortures to which this Man was subjected. An event that influenced the rapid course of the Passion and the cause of death of the TS Man was the fall under the weight of the cross. This Man shows, on the right side, shoulder lowering, flat hand and henophthalmos, revealing a violent blunt trauma, from behind, to neck, chest and shoulder, with the entire brachial plexus injury and muscular damage to the neck bottom with the head bent forward and turned to the left, on the cross, as he had a stiff neck. Most likely, falling the body forward, the chest trauma caused a heart and lung contusion with hemothorax. The wrists were easily nailed in the Destot space with ulnar artery cutting and partial tear of the ulnar nerve cause of the thumb retraction and its disappearance on the Shroud. The nail in the right foot was driven in the tarsal bones. The right foot was probably dislocated to the ankle. The lance penetrated in the sixth intercostal space. The likely cause of immediate death was a myocardial infarction with rupture, haemopericardium and heart tamponade of a subject with cardiac contusion. Tortures and other medical conditions have only accelerated the death.
\end{abstract}

Keywords: Turin Shroud, trauma, humerus and ankle dislocation, Hands and feet nailing, Lung and cardiac contusion.

\section{Introduction}

The TS [1,2] is a linen cloth, $4.4 \mathrm{~m}$ long and $1.1 \mathrm{~m}$ wide, which wrapped a dead body with evident rigor mortis [3] of a scourged, thorn-crowned man who was crucified and stabbed in the side with a spear.

Many medical aspects regarding the Passion of the TS Man remain unclear even if a striking congruence between the TS Man and Jesus Christ described in the Gospel can be detected. For example, it is not simple to explain thoroughly the posture of the hands or the point of penetration of the nails in the hands. 
His immediate cause of death is not simple to be detected from the analysis of the body image and the diagnosis of asphyxia and/or severe cardiovascular collapse proposed by many [3] as a probable cause of death for a crucified man is inconsistent with the Gospel, which states that Jesus was in perfect lucidity and He has had the strength to cry out immediately before his death.

In order to spread more light about some uncertain medical aspects we undertook a cross-disciplinary study involving medical and engineering research about certain aspects of the TS that have so far been neglected, or only superficially explored. In particular we have studied:

- the upper limbs posture (the position of the right hand that touches the outer edge of the left thigh, the crossing of the hands on the pubis, the absence of the thumbs);

- $\quad$ the II, III, IV, V right fingers posture: the extended fingers; the posture of II, III, IV, V left fingers: the flexed last phalanges);

- $\quad$ the head and trunk posture (the head bending, the right shoulder lowering);

- $\quad$ the right eye retraction significance;

- $\quad$ the left hand and right foot nailing;

- the blood draining origin and significance (blood on the head, forearms and feet; the large stain of blood clots and serum from the right chest).

Finally, we posed the question whether Jesus carried the cross or the patibulum and if his body was washed after the deposition.

The answers to all these questions have been recently published in, or submitted to, international journals $[4,5,6,7,8]$ and are summarized here in the present paper.

\section{Experimental procedures on limbs}

We performed our studies with the images of the TS and experiments on cadaver limbs.

Many photographs have been used also in digital form and the corresponding image details have been compared together [6]. The position of the TS Man was reconstructed by a digital processing based on the overlapping of a manikin of a numerical TS. Moreover, a detailed study had been performed on high-resolution images of the TS, comparing results with typical anthropometrical indexes of a man [6].

Anatomical analyses were performed on three right upper limbs resected from cadavers at the shoulder, and another anatomic specimen consisting of the trunk and both inferior limbs [5]. The donors gave specific consent. Cadavers were managed by the Body Donation Programme of the Section of Human Anatomy of the Department of Molecular Medicine of the Univeresity of Padua (according to European, National and Regional normative references $[9,10]$.

The upper limbs were nailed in the following points:

- $\quad$ radiocarpic joint between the radio, lunate and scaphoid;

- $\quad$ midcarpal joint line between the scaphoid/lunate and trapezoid/capitate;

- midcarpal joint between the lunate/pyramidal and capitate/uncinate (Destot's space) by nailing at the median skin-fold of the wrist, just above and medially to the pisiform;

With regard to the inferior limbs, the following procedures were performed:

- dislocation of the right joint, through increasing weights applied with a rope to the ankle (tension technique usual in the crucifixion);

- $\quad$ nailing of the left foot between $1 \mathrm{st}$ and 2 nd metatarsal bone, with a $10 \mathrm{~cm}$-long nail;

- nailing of the left foot between the scaphoid and cuboid, proximally, and third cuneiform, distally, with a $25 \mathrm{~cm}$-long nail;

- $\quad$ further nailing of the right foot, with the long nail passing through the left one, at the level of the right tibiotarsal joint.

Points of entrance were identified under radioscopic control; CT (Computed Tomography) axial scans of the specimens were acquired; specific anatomical dissections were then performed to confirm the CT findings and to verify the condition of vessels and nerves. 


\section{Results and comments}

On the shoulders two heavier contusion areas are identifiable [7] not much compatible with carrying a pair of beams, stipes and patibulum tie together [Fig. 1].

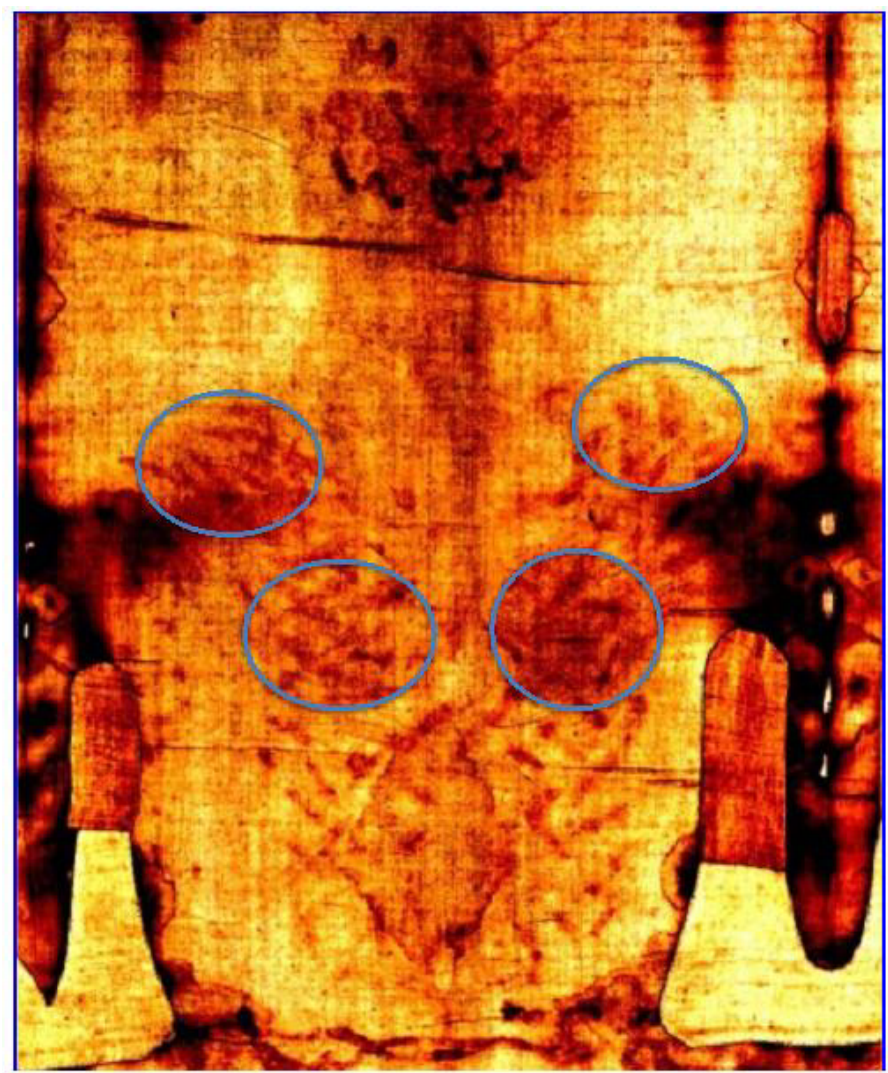

Figure 1. The high-contrast upper dorsal image of the TS highlights two pair of bruises caused most likely by the cross brought on the right and left shoulder.

It is therefore much more likely that the TS Man has carried the cross rather the patibulum [11]. If it were so, it would mean that the Man of the Shroud has carried the cross on both shoulders, first right and then left. It can therefore reasonable be assumed that:

1. the more marked bruise on the left shoulder would be due to the higher load on this area in a more curved subject because weakened by the previous fall;

2. the TS Man has fallen more than once.

The authors believe that the fall under the weight of the cross has been an event, so far underestimated, which has influenced the rapid course of the TS Man Passion. The reconstruction of events is that the Man beating the chest to the floor, unable to defend himself putting his hands on, suffered a serious trauma to the neck with neuromuscular damage, to the shoulder with humerus dislocation, and to the chest with lung and cardiac contusion. Likely, the lung contusion caused bleeding into the pleural cavity with hemothorax, poured out with the post mortem spear.

The arms bent at the elbows about 45 degrees, despite the rigor mortis (on the cross, his arms were stretched out and their angle with the body was about $15^{\circ}$ ) indicate that the hands were reassembled on the pubis and they were not disjointed because the right hand was hooked by the bent and stiff fingers of the left hand. Indeed, on TS there are not imprints compatible with bindings. 
The crossing of the hands on the pubis, and not above it, in a person with hyperexpanded chest and erected trunk, certainly not in ventral flexion, is consistent with a crucified man who has undergone a stretching of the arms during the nailing and while he was hanging on the cross at the wrists.

Measures of the back aspects of various segments of the arms, from the hands up to the shoulders, show that the right humerus is below the shoulder of about $3.5 \mathrm{~cm}$. The abnormal position of the right hand, too elongated, which reaches the outer edge of the left thigh, confirms our hypothesis.

The hands posture of the TS Man differs from that considered characteristic, the "crucified clenched", with complete failure of flexion of the thumb and index finger and with partial failure of flexion of the middle finger ("blessing hand") by proximal paralysis of the median nerve at the elbow/proximal forearm, as a consequence of prolonged upper extremity abduction, extension, and external rotation on the cross [12]. So the different posture of both hands as to the typical crucified clenched is to assume that di TS Man has suffered nerve damages before nailing in various ways, both in the right arm and in the left.

The right hand posture with flat four fingers cannot depend by the rearrangement of the corpse because the initial contracture would be come back, as it is known in forensic medicine; so we too must exclude that a so strong man and in rigor mortis could be positioned with a lowered shoulder by about $15^{\circ}$. Also the right enophtalmos can be attributed to a nervous injury rather than to right cheekbone swelling effect. Everything is explained according to a serious trauma, from behind at the base of the neck. It had as consequences: paralysis of the entire brachial plexus and, then, of the right arm (dangling, intra-rotated by dislocation); cervical sympathetic paralysis (Claude-Bernard-Horner Syndrome); muscle damage (represented in the Christian Tradition with the iconography of the Crucifix with the head bent forward and turned to the left, as he had a stiff neck, due to the contraction of the unhurt sternocleidomastoideus muscle. If it was so, it is reasonable to infer that, despite the head of the deposed Man was easily straightened because injured muscles, a slight deviation of the neck to the right by about $10^{\circ}$ remained.

The left hand posture is suggestive of a "claw hand", probably due to stretching until the dislocation of the arm caused by ropes during the nailing, with proximal lesion of the ulnar nerve. However, a claw hand is also characterized by abduction and extension of the thumb, which is not evident in the TS. This contradiction can be explained considering that the nailing in the Destot space, as in our experiment, has involved still intact and excitable fibers of the ulnar nerve, in particular its deep branch, with contraction of the adductor pollicis and flexor pollicis brevis muscles.

The thumb retraction was achieved either by nailing in the radio-carpic space (precisely between the tendon prominences of the flexor radialis carpi and the palmaris longus muscles, in the median skinfold of the wrist near the scaphoid tubercle) with involvement of the flexor longus pollicis tendon and median nerve, or by nailing in the Destot space near to the pisiform bone, with involvement of the ulnar artery and nerve.

The thumb retraction was obtained by P. Barbet [3], nailing in the centre of the main skin-fold (the middle) causing a partial laceration of the median nerve in the ulnar side, likely saving the fibers that innervate the thumb muscles. B. Lussiez [13], nailing the same site, found a median nerve deviation to the radial side. So, there are various possibilities of nailing without bone fractures, that cause equally thumb retraction. According to the tests made by authors [7], the more likely nailing in the TS Man case was in the Destot space: the lesion of the ulnar artery could explain the post mortem bleeding on the left wrist and forearms, due to unnailing.

In this regard, the authors believe that the body of the TS Man has been washed or at least cleaned. The blood on the TS would be post mortem coming from the lance to the chest, by the removing the helmet of thorns and by the unnailing; they hope in further studies to document any vascular and nervous lesions at the level of the nail outlet hole.

The analysis of the right footprint leads to two conclusions:

1. The nail was located at $45 \%$ of the entire foot sole, starting from the heel, consistent with a nailing in the tarsal bones, not in metatarsal bones, probably among the navicular, cuboid and intermediate cuneiform. This nailing did not caused fractures in our experiment.

2. The right foot has suffered an ankle dislocation, as fingerprints are still present and in this condition, experimentally, their distance from the table is about $5 \mathrm{~cm}$. If the front part of the foot was 
raised as normally, it would be too distant from the sheet and therefore not imprinted. The authors believe that the Shroud was lying over a "mattress" of powered myrrh, aloe and natron, on which the body of the Man was laid; then, turning around his head, it was rested on his body, without being tied. In agreement with N. Miani [14], the spear struck the sixth intercostal space, not the fifth. The wound on the side, clearly imprinted in the Shroud, is located about $2 \mathrm{~cm}$ below the edge of the pectoral muscles, which normally are well developed down to the fifth intercostal space. The roman spearhead, maximum $5 \mathrm{~cm}$ wide (consistent with the chest wound, $4.5 \mathrm{~cm}$ wide) and about $28 \mathrm{~cm}$ long, could have easily pierced even the pericardium, which is about $12 \mathrm{~cm}$ from the chest, so the spilled blood would have been originated either from the pleural cavity or the endopericardium.

After the analyses performed on the TS and the comparison of these results with the description of Jesus' Passion reported in the Gospels, the authors believe that the TS Man is Jesus of Nazareth. The cardiac contusion, characterized by areas of muscular waxy necrosis, accelerated the course of the Passion, ended with myocardial infarction, heart rupture and hemopericardium with cardiac tamponade. These were the immediate cause of death after issuing a cry, in perfect clarity, as it appears in the Gospels. Tortures and other conditions, such as traumatic-hemorrhagic-hypovolemic shock, ventilatory failure and causalgia, have only hastened it.

\section{Acknowledgments}

The authors warmly thank prof. Giulio Fanti, Associate Professor - Department of Industrial Engineering - University of Padua, who performed the processing of high-resolution images of the Shroud and participated in the experiments on cadavers. Without him, this paper would not have been possible.

\section{Conflict of interest}

The authors have no conflicts of interest actual or potential to declare.

\section{References}

1. M. Antonacci, Resurrection of the Shroud: New Scientific, Medical, and Archeological Evidence M. Evans \& Co, New York (2001)

2. G. Fanti, P. Malfi, The Shroud of Turin, First century After Christ Pan Stanford Pub. Singapore (2015)

3. P. Barbet, A Doctor at Calvary: the passion of our Lord Jesus Christ as described by a surgeon The Earl of Wicklow (trans), Garden City, NY: Image Books Ed, 116 (1963)

4. M. Bevilacqua, G. Fanti, M. D’Arienzo, R. De Caro, Injury 45, 460 (2014)

5. M. Bevilacqua, G. Fanti, M. D’Arienzo, A. Porzionato, V. Macchi, R. De Caro, Injury, Suppl 6, S142 (2014)

6. M. Bevilacqua, G. Fanti, M. D’Arienzo, R. De Caro, Injury 46, 2074 (2015)

Reply to: V.L. Caja, M.M. Reverte-Vinaixa, Injury 45, 1804 (2014)

7. M. Bevilacqua, G. Fanti, M. D’Arienzo, (submitted for publication)

8. M. Bevilacqua, G. Fanti, M. D’Arienzo, (submitted for publication)

9. R. De Caro, V. Macchi, A. Porzionato, Anat. Sci. Educ. 2, 91 (2009)

10. A. Porzionato, V. Macchi, C. Stecco, A. Mazzi, A. Rambaldo, G. Sarasin, et al. Anat. Sci. Educ. 5, 264 (2012)

11. C. Glori, Proc. Int. Workshop IWSAI, ENEA Frascati, Italy, (2010)

12. J.M. Regan, K. Shahlaie \& J.C. Watson, Brain and Behavior 3, 243 (2013)

13. B. Lussiez, Chirurgie de la main 24, 132 (2005)

14. G. Ricci. L'Uomo della Sindone è Gesù (Studium Ed, Italy, Rome, 401, 1969) 